\title{
Cardiac Ballet: Repetitions of Complex Electrocardiographic Patterns
}

\author{
F. H. SMIRK AND J. NG \\ From Wellcome Medical Research Institute, Department of Medicine, University of Otago Medical School, Dunedin, \\ New Zealand
}

During the past 12 years 7 examples have been encountered of brief arrhythmic episodes which we have called the "cardiac ballet". They consist of sequences of multiform ventricular (QRST) complexes, with or without ventricular complexes of the shape of sinus beats interspersed. The special feature of the arrhythmia is that the characteristic patterns of the sequences are from time to time replicated.

All of our 7 examples of this condition have been associated with the $R$ on $T$ phenomenon (Smirk, 1949; Smirk and Palmer, 1960; Ng, 1968), and in one case an episode of ventricular fibrillation was recorded.

Another recurrent ventricular arrhythmia is "repetitive paroxysmal ventricular tachycardia", which has been described by Parkinson and Papp (1947), Katz and Pick (1956a), and Stock (1962), but almost all their published traces differ from the episodes we are about to describe. Further, Parkinson and Papp (1947) drew attention to the generally good prognosis in repetitive paroxysmal ventricular tachycardia, whereas 5 out of our 7 patients died.

\section{METHODS}

Fig. 1, 3, and 6 were obtained from long recordings of one or more leads of the standard 12-lead electrocardiogram. Fig. 2, 4, 5, and 7 were obtained during prolonged electrocardiographic monitoring, using scalar leads of the cube vectorcardiographic reference system (Grishman and Scherlis, 1952). These traces were recorded on magnetic tape and then transferred onto paper as described by Wallis, Meek, and Ng (1968).

\section{RESULTS}

The electrocardiographic traces in each of Fig. 1-7 have been arranged one below each other so

Received October 25, 1968 as to illustrate the manner in which the sequences of the various ventricular complexes have been replicated. Some of the traces are parts of continuous records, but other traces may have been recorded at time intervals as far apart as hours. Sometimes, the replication has involved almost exact repetition of an electrocardiographic pattern; in other instances there were minor variations but the general sequence has been preserved.

A case history follows each Figure and legend. As yet there do not appear to be clinical characteristics which warrant subgrouping of the patients.

\section{CASE Reports}

Case 1. A young woman, 19 years of age, five months pregnant, who had mitral stenosis and pulmonary venous congestion, was treated by a closed mitral valvulotomy operation. Before operation she was digitalized and given a mercurial diuretic. Normal rhythm was recorded in a pre-operative electrocardiogram.

During operation, before the pericardium was opened, the arrhythmia occurred (Fig. 1) which was responsible for our use of the term "cardiac ballet". The recurring sequences of four or five ventricular complexes developed after atrioventricular dissociation which involved independent sinus and apparent atrioventricular nodal impulses. A short run of ventricular tachycardia also occurred after a $T$ wave interruption.

Normal rhythm returned quickly after valvulotomy was performed.

Case 2. A woman, 57 years of age, had angina pectoris for 10 years, previous hypertension, an extension of a past infero-lateral myocardial infarction, left ventricular failure, attacks of acute pulmonary oedema, hypotension, and frequent cardiac ischaemic pain. She received digoxin, $0.25 \mathrm{mg}$. daily, and diuretic therapy, but was on no antiarrhythmic drugs for several days before death. The serum potassium was $4 \cdot 1 \mathrm{mEq} / 1$. and the blood urea was $42 \mathrm{mg}$. $/ 100 \mathrm{ml}$. on the day before death. Two attacks of ventricular fibrillation occurred 426 


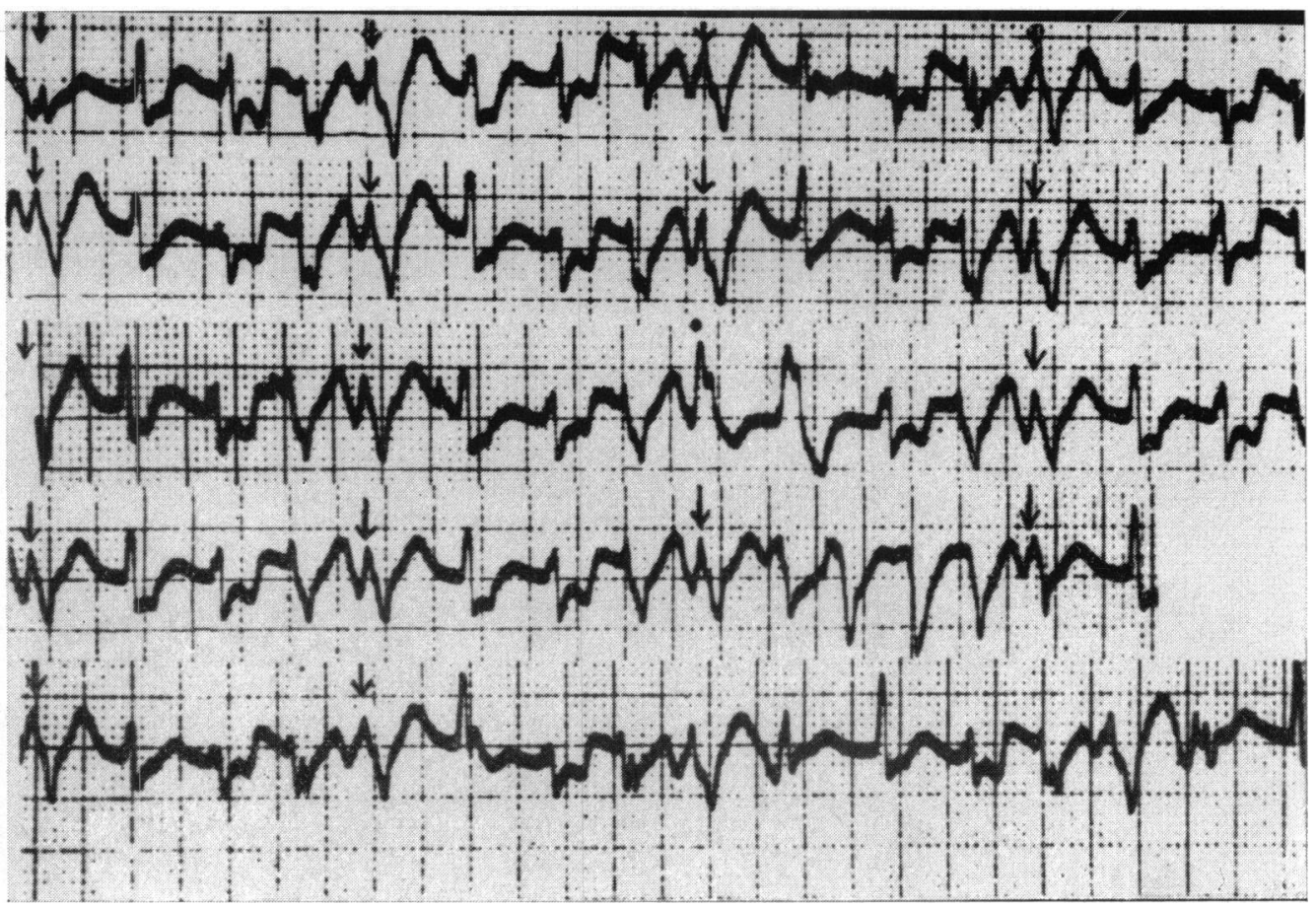

FIG. 1.-Case 1. Five strips of a lead II trace are shown. Recurring sequences of four ventricular complexes are seen in the top four strips, the end of such sequences being marked by arrows. The bottom strip shows a sequence of four ventricular complexes which is followed by two sequences of five ventricular complexes. The individual ventricular complexes which make up the sequences differ in shape and all differ from the form of the characteristic ventricular complex of sinus beats recorded before operation in the same lead. In the middle strip, a pair of ventricular ectopic complexes (the first complex of the pair is indicated by a dot) occurs interposed among the recurring sequences. A $T$ wave interruption occurs in the fourth strip from the top involving the $T$ wave of the ventricular complex under the third arrow; it is associated with a short run of ventricular tachycardia.

attack and sudden death from the second. The cardiac ballet rhythm preceded the onset of the first attack of fibrillation (Fig. 2) but not the second attack; examples of $T$ wave interruptions occurred before and at the start of both attacks of ventricular fibrillation. Runs of ventricular tachycardia of three or more complexes also occurred in the 24 hours before death, but none of these paroxysms occurred during the cardiac ballet rhythm. A necropsy confirmed the recent and old myocardial infarcts.

Case 3. An elderly man of $\mathbf{7 4}$ years had bronchopneumonia, ischaemic heart disease, and mild diabetes mellitus. He was very dyspnoeic on admission and numerous ventricular premature beats were present, together with a run of four multiform ventricular complexes (bottom trace, lead V3, Fig. 3). The upper four traces of Fig. 3 show the cardiac ballet rhythm, the $R$ on $T$ phenomenon, and another run of four multiform ventricular complexes, and were obtained from a V2 strip recorded 18 hours after admission when his clinical state had only slightly improved. Ventricular fusion complexes are well seen in these four traces. The serum potassium was $3.5 \mathrm{mEq} / 1$. and the blood urea $38 \mathrm{mg} . / 100$ $\mathrm{ml}$. about the time of the second electrocardiogram.

Substantial recovery was evident some 30 hours after admission. Propranolol was started then, but the arrhythmias were already abating with his general improvement. He had no digitalis or diuretic therapy at home or in hospital up to this time, and sympathomimetic therapy was not charted until the third day of admission. No ectopic beats were found in routine electrocardiograms by the fifth day of admission. Later in the same hospital admission, during another bout of pneumonia and while still on propranolol, he developed a myocardial infarction which was seen in the electrocardiogram to be of a partial thickness anterior type, but few ectopic beats were recorded. His family doctor reports that for the two years after these events he has kept relatively well.

Case 4. An elderly man of 76 years was admitted in congestive heart failure with hypokalaemia (serum potassium $2.3 \mathrm{mEq} / \mathrm{l}$.), low blood pressure, and mesenteric artery thrombosis. The blood urea was $82 \mathrm{mg} / 100$ $\mathrm{ml}$. on admission. Previously he had hypertension, 


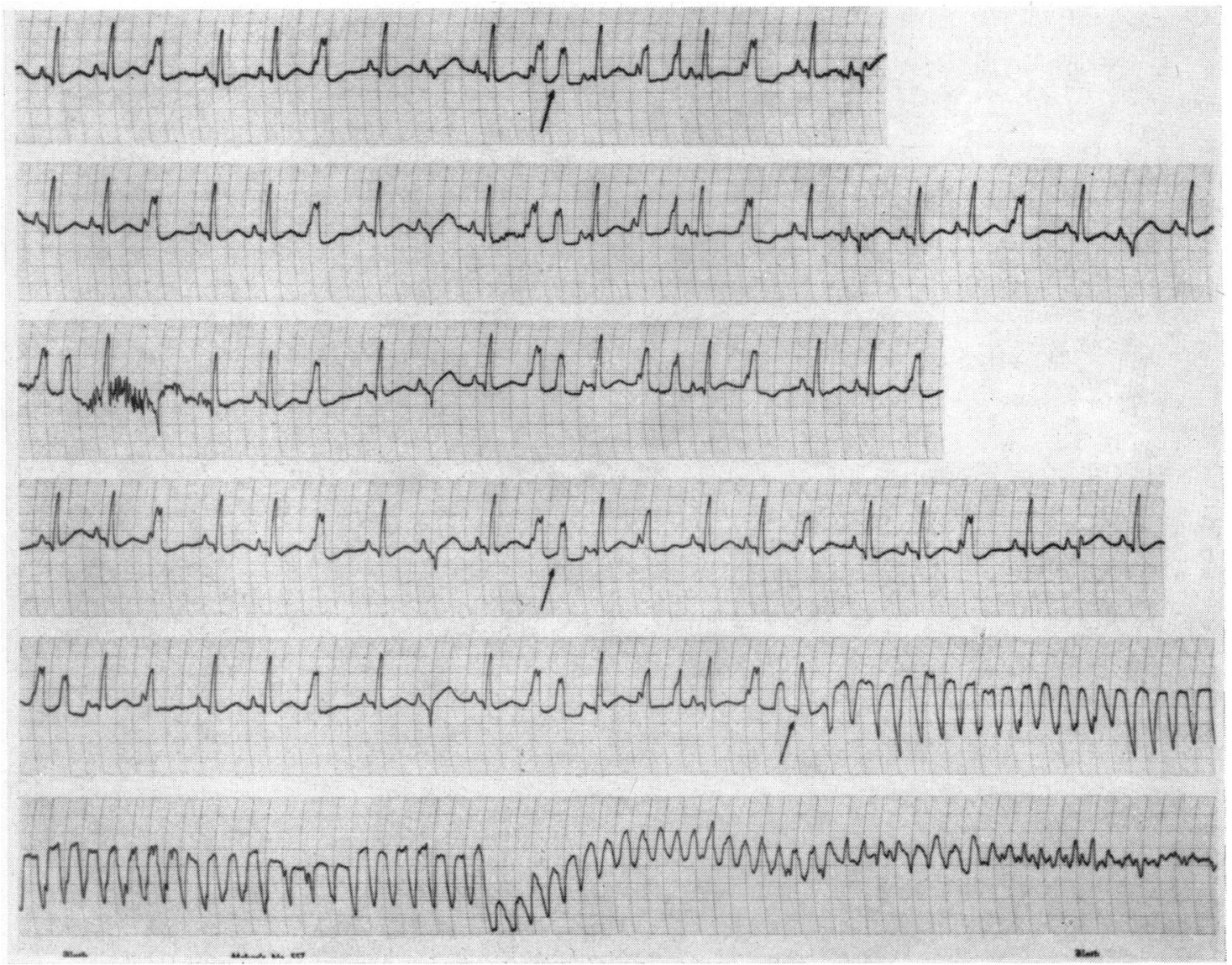

Fig. 2.-Case 2. The six strips show a continuous cube system scalar lead A record. The cardiac ballet rhythm began in the top strip. The four upper strips show two principal types of abnormal ventricular complexes, e.g. the third and eighth ventricular complexes in the top strip. Examples of $T$ wave interruptions caused by ventricular ectopic complexes are indicated by arrows. The onset of ventricular fibrillation is shown in the fifth strip from the top, and its further development is evident in the bottom strip.

congestive heart failure, intermittent claudication, a history of an anterior myocardial infarction, and electrocardiographic evidence of an old inferior myocardial infarction. He had been receiving digoxin $0.25 \mathrm{mg}$. daily until this was stopped 3 days before admission because of anorexia and vomiting, but he continued to take two tablets daily each consisting of $0.25 \mathrm{mg}$. cyclopenthazide and $600 \mathrm{mg}$. potassium chloride. Fig. 4 shows an atrial tachycardia with second-degree atrioventricular block, the cardiac ballet rhythm, and $T$ wave interruptions. A long tape recording of his electrocardiogram at this time showed numerous similar recurring sequences.

The atrial tachycardia ceased and the ventricular ectopic systoles were diminished by about $20 \mathrm{mEq}$ potassium chloride intravenously administered before laparotomy and small bowel resection. The patient gradually deteriorated, and died 24 hours after operation. After operation he was on no antiarrhythmic drug apart from intravenous potassium supplements, and was given digoxin $0.25 \mathrm{mg}$., but an electrocardiogram taken three and a half hours before death showed normal rhythm. The serum potassium about 5 hours after operation was $2.9 \mathrm{mEq} / 1$. A necropsy revealed healed anterior and inferior myocardial infarcts.

Case 5. A woman aged 62 years had mild hypertension in the past, and was admitted with a full-thickness anterior myocardial infarction. When seen at home she was said to be almost pulseless but on arrival at hospital the blood pressure was $115 / 60 \mathrm{~mm} . \mathrm{Hg}$ and maintained itself about this level.

She was in normal rhythm on admission; the blood urea was $37 \mathrm{mg} . / 100 \mathrm{ml}$. and the serum potassium was $4.5 \mathrm{mEq} / 1$. Three hours after admission, when she was asymptomless and not in heart failure, she developed what appeared at first to be chaotic heart rhythm.

A tape recording of her electrocardiogram at this time 


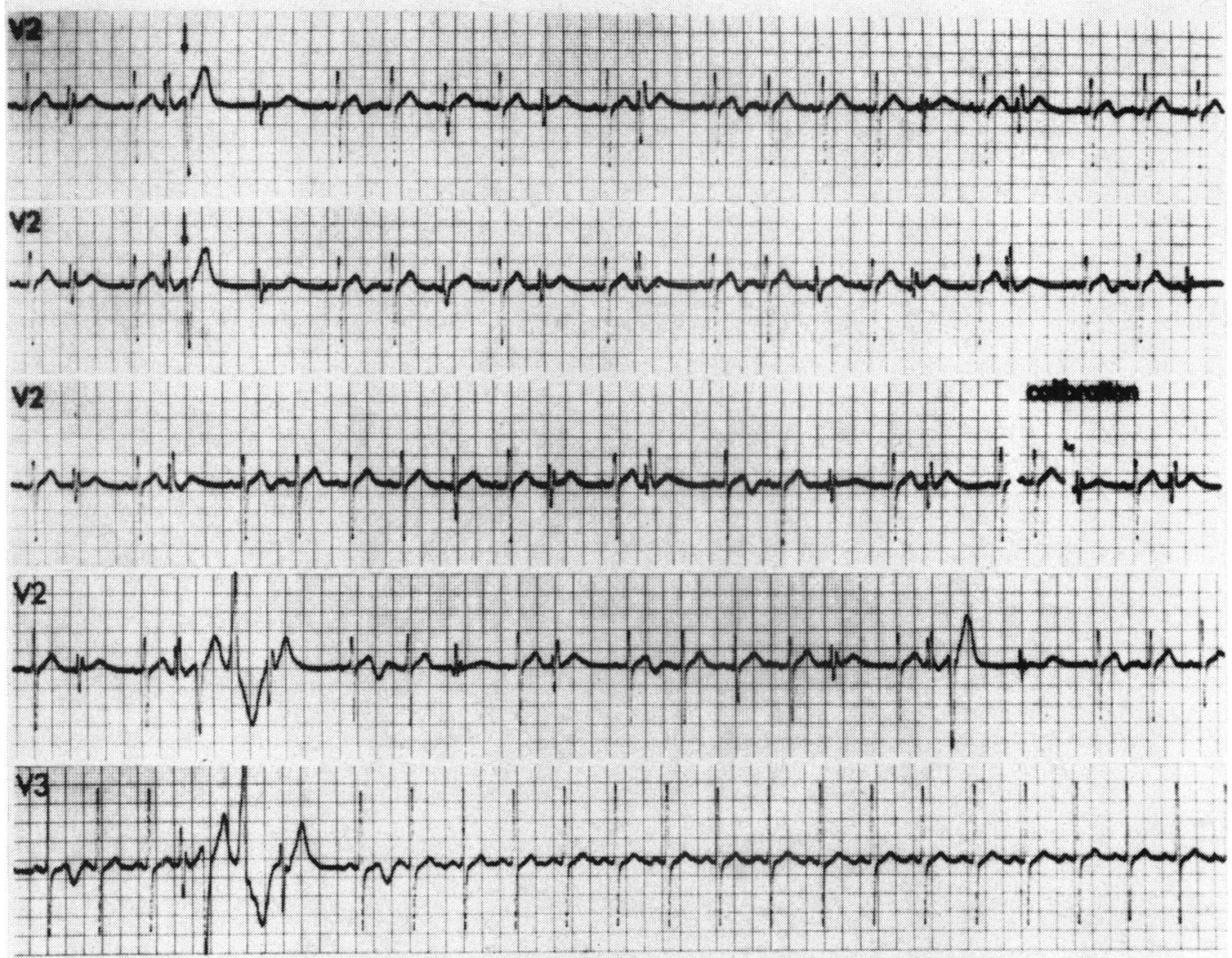

FIG. 3.-Case 3. The upper four traces are of a V2 record, and display recurrent sequences of 7 to 9 intermixed multiform and normal ventricular complexes. The arrows mark two of the several $T$ wave interruptions present; the interruptions occur early on the T wave. The bottom trace is of a lead V3 strip, and was recorded 18 hours earlier than the upper traces. The run of four multiform ventricular complexes in this bottom trace shows some resemblance to the run recorded in the fourth trace from the top.

showed intermittent atrioventricular dissociation involving sinus and apparent atrioventricular nodal impulses, the latter showing aberrant ventricular conduction. Frequent multiform ventricular ectopic systoles, repetitive sequences of various ventricular complexes, $\mathrm{T}$ wave interruptions, and short paroxysms of ventricular tachycardia were also recorded. Fig. 5 shows eight examples of a recurring pattern (these are arranged in a non-chronological order), a short run of ventricular tachycardia, and probable atrioventricular dissociation.

The tape recording of this patient's electrocardiogram was not kept, but parts of the recording were transferred onto paper. These paper records showed another 20 sequences resembling the examples of Fig. 5 when scanned over the same period of time (some 6 hours).

Normal rhythm was restored by procainamide first given intramuscularly and then orally administered, but she needed up to $3 \mathrm{~g}$. daily. Four weeks after admission she still required procainamide daily in four oral doses of $250 \mathrm{mg}$. because of occasional ventricular ectopic beats. She received no digitalis therapy in hospital, but before admission she was taking daily digoxin 0.25 mg. and two tablets each containing $0.25 \mathrm{mg}$. cyclopenthiazide, $0 \cdot 1 \mathrm{mg}$. reserpine, and $600 \mathrm{mg}$. potassium chloride. She "dropped dead" while gardening five months later. No necropsy was performed.

Case 6. A man aged 59 years was admitted in a very ill state from severe emphysema and gross cor pulmonale. He had hypertension in the past, but there was no known history of ischaemic heart disease. The electrocardiogram on admission showed numerous ventricular ectopic complexes and a paroxysm of ventricular tachycardia.

Fig. 6 was obtained two days after admission and shows recurring sequences of four ventricular complexes. A $\mathrm{T}$ wave interruption was seen in another lead of this electrocardiogram.

The serum potassium was $3.8 \mathrm{mEq} / 1$. on admission and $3.4 \mathrm{mEq} / \mathrm{l}$. on the third day after admission; the 

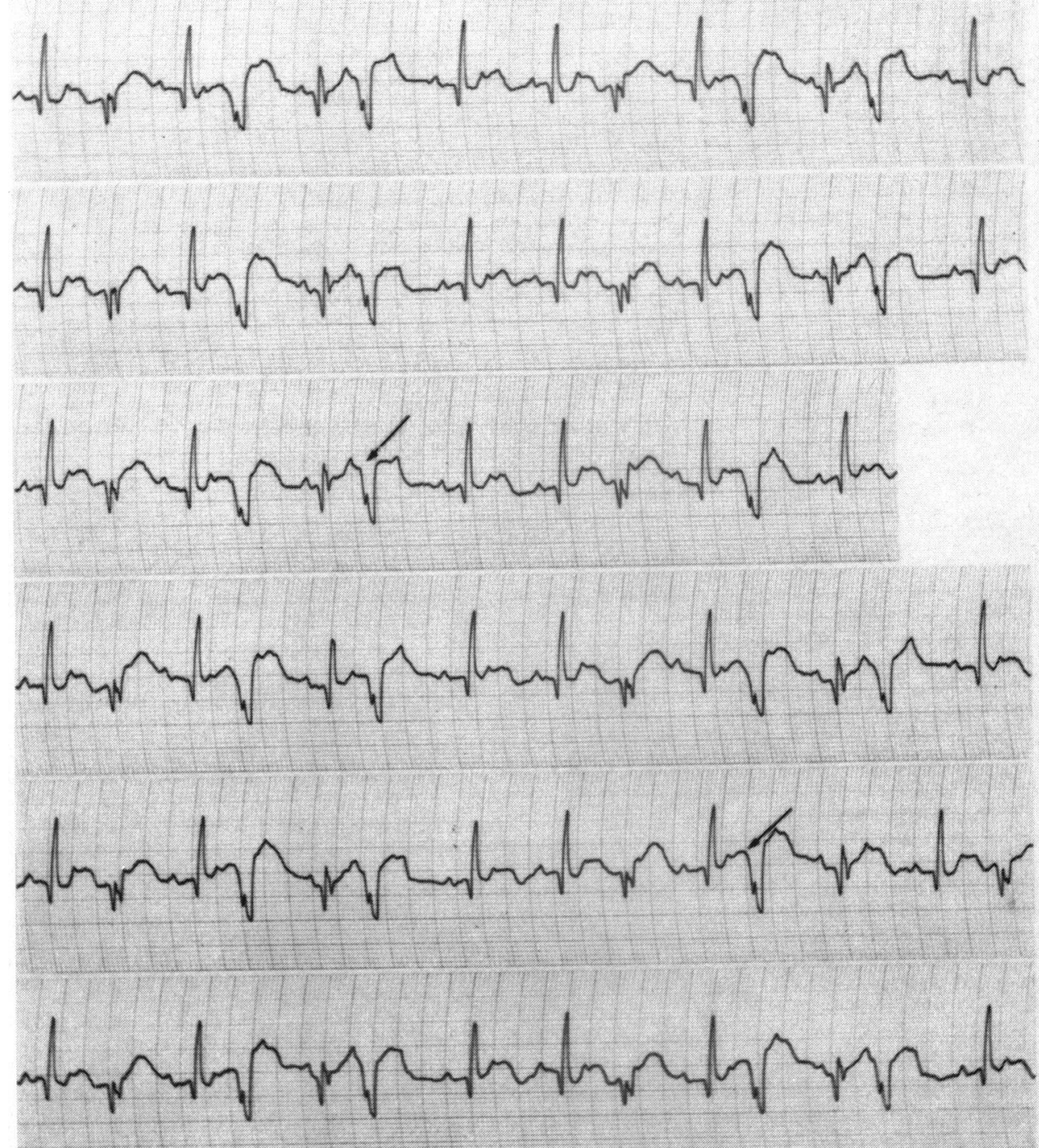

Frg. 4.-Case 4. The strips are of a cube system scalar lead A record; the third and fourth strips from the top are continuous. An atrial tachycardia (rate $150 / \mathrm{min}$.) is associated with a basic 2:1 atrioventricular block. Recurrent sequences occur of 11 to 14 intermixed multiform ventricular ectopic complexes and ventricular complexes of the supraventricular beats. The second and fourth ventricular complexes of the upper strip represent the two main forms of ventricular ectopic complexes present; the fifth and twelfth ventricular complexes of the same strip may be ventricular fusion beats. The arrows point to two of several $T$ wave interruptions.

corresponding blood urea levels were over $200 \mathrm{mg}$. and $92 \mathrm{mg}$., respectively. His therapy at home had included digoxin $0.25 \mathrm{mg}$. daily, procainamide, and one tablet containing $0.25 \mathrm{mg}$. cyclopenthiazide and $600 \mathrm{mg}$. potassium chloride on alternate days. The last drug was replaced 8 days before admission by one tablet of frusemide $40 \mathrm{mg}$. twice daily, apparently without potassium supplements. The procainamide was dis- continued in hospital, and digoxin and sympathomimetic drugs were withheld until a week after admission, when the ventricular ectopic beats were occurring much less frequently. He remained on diuretic therapy with oral potassium chloride supplements.

The patient died suddenly 45 days later during the same hospital admission. A necropsy revealed right and left ventricular hypertrophy and substantial coronary 


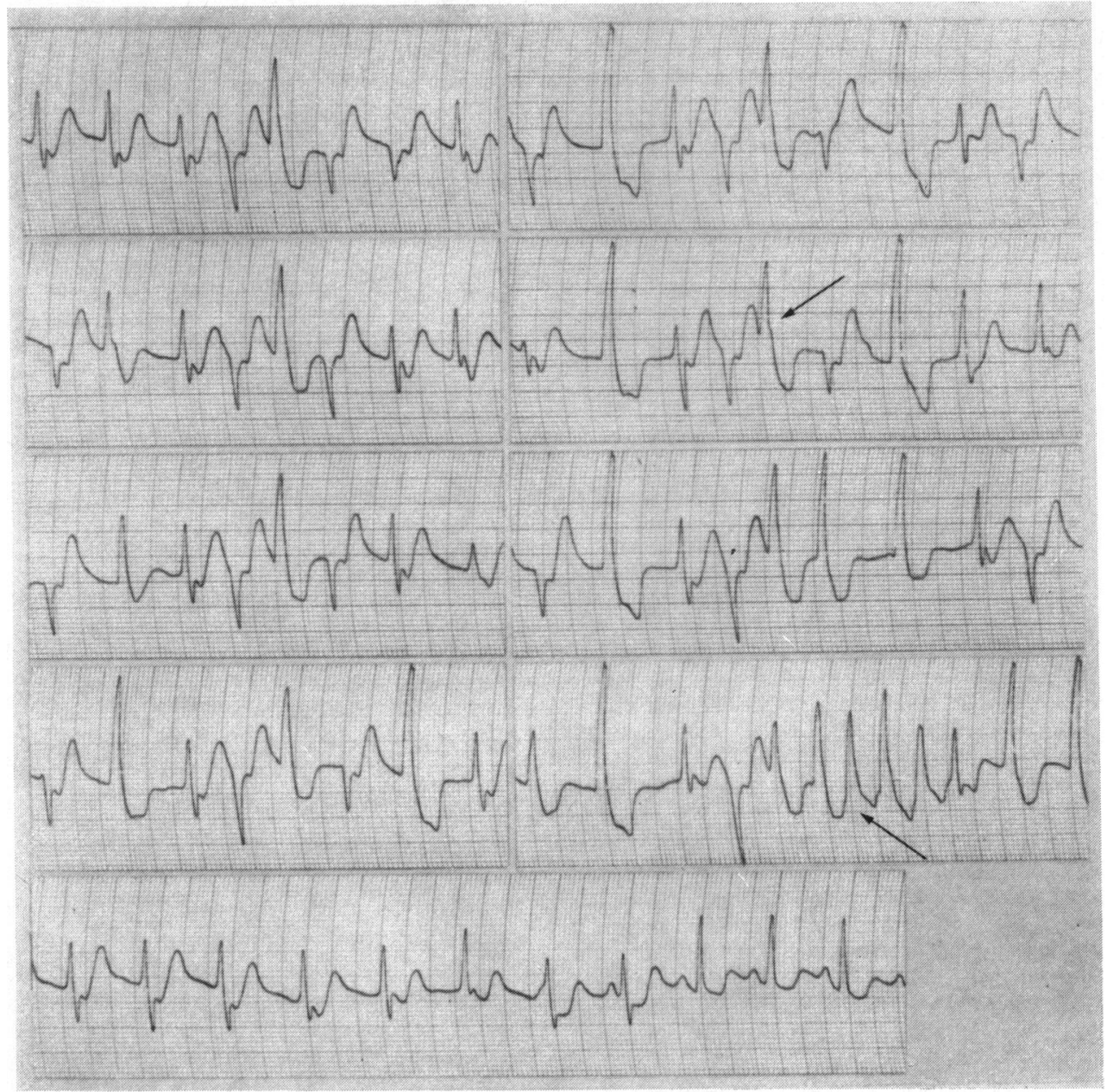

Fig. 5.-Case 5. The nine traces are of a cube system scalar lead $\mathrm{C}$ record. The third, fourth, and fifth ventricular complexes of each of the upper eight traces form a characteristic sequence; in addition, the second ventricular complex of five traces and the sixth ventricular complex of six traces resemble each other. A short run of ventricular tachycardia follows the fifth ventricular complex in the fourth right-hand trace from the top. The bottom trace, is a continuation of the fourth left-hand trace from the top and shows a probable example of atrioventricular dissociation; three sinus beats occur at the end of this trace. The arrows point to two of the several $T$ wave interruptions present.

atherosclerosis, but the myocardium appeared normal macroscopically and histologically.

Case 7. An elderly woman of 70 years had previous hypertension (retinal grade 2 Keith-Wagener changes), angina pectoris for five years, congestive heart failure, atrial arrhythmias, and increased frequency and severity of cardiac ischaemic pain.

During a month-long hospital admission, she had a recurrence of heart failure at a time when the serum potassium was $2.8 \mathrm{mEq} / \mathrm{l}$. and the blood urea was 160 mg./100 ml. Digoxin ( $0.25 \mathrm{mg}$./day) had been stopped for 24 hours 2 days previously because of vomiting. Electrocardiographic monitoring showed an atrial tachy- cardia with multiform $\mathbf{P}$ waves and atrioventricular block, frequent multiform ventricular ectopic complexes, $T$ wave interruptions, and short runs of ventricular tachycardia. The traces of Fig. 7 were recorded in the course of one minute; each trace shows similarities to the other two. No other such examples occurred.

The patient's arrhythmic state improved with the cessation of digoxin again and the oral administration of potassium chloride and procainamide. The next day, however, she developed severe ischaemic pain and low blood pressure, and a week later she died suddenly from ventricular fibrillation. The terminal electrocardiogram was recorded and showed numerous $T$ wave interruptions and short runs of three or four ventricular 


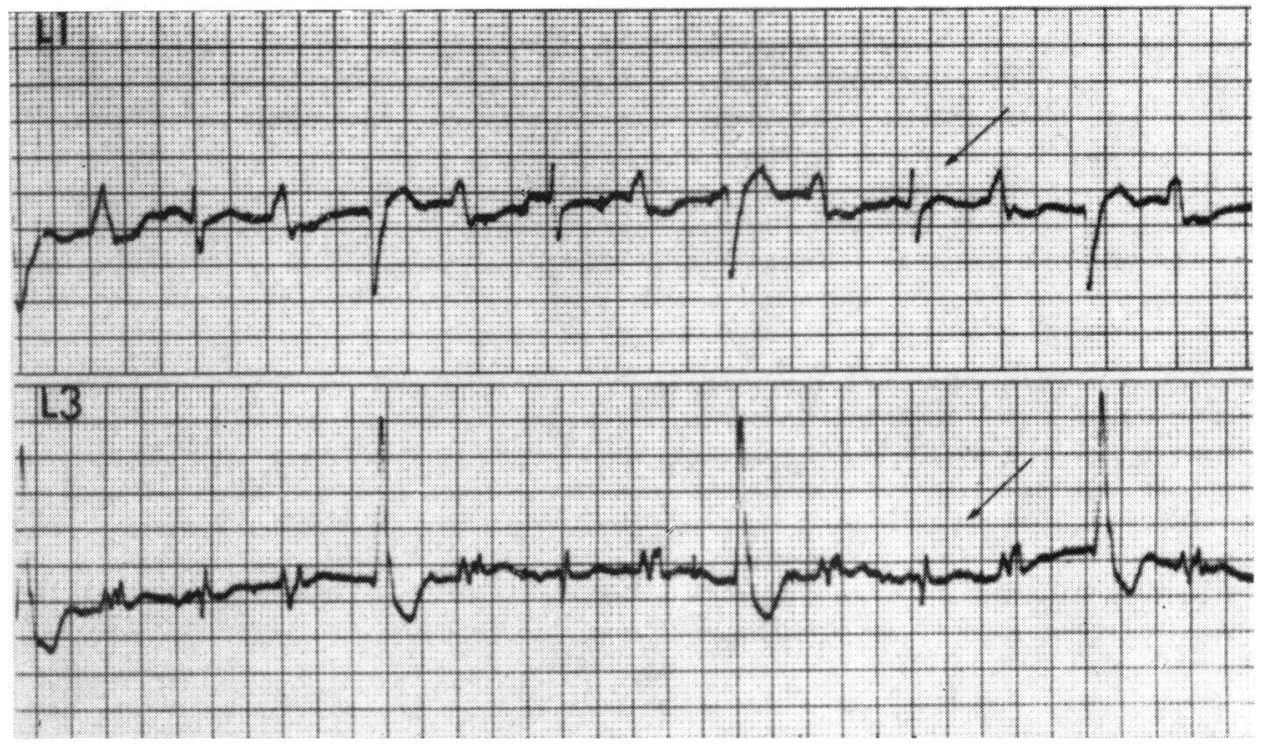

Fig. 6.-Case 6. The upper and lower traces are of lead I and lead III records, respectively. They show recurring sequences of four ventricular complexes. The arrows point to ventricular complexes of the usual contour in these leads.

ectopic complexes before ventricular fibrillation occurred, but no cardiac ballet. A necropsy, which was restricted to the heart, showed the thickness of the left ventricular wall at the base of the heart to measure $2 \cdot 0$ $\mathrm{cm}$. The anterior descending, circumflex, and right coronary arteries were widely patent. No recent myocardial infarct was found, but several large focal areas of fibrosis, thought probably to be due to ischaemia, were demonstrated histologically.

\section{Discussion}

The central feature of the arrhythmia we have described as the cardiac ballet is the repetition of a series of multiform ventricular complexes. It already seems likely that there is more than one way in which such a recurrent arrhythmia may arise, and in this circumstance the non-technical nature of the term "cardiac ballet" appears appropriate.

The multiform ventricular complexes in this arrhythmia may have two or more different ventricular foci of origin. However, we prefer the term multiform to multifocal, because Palmer (1962) showed experimentally that when the rate of stimulation of a single region of the ventricles is increased progressively, the complexes of the ventricular responses become multiform, presumably because the pathways of conduction of the complexes vary from one to another. Multiform ventricular complexes in the cardiac ballet rhythm may arise also from aberrant ventricular conduction of supra- ventricular impulses and from ventricular fusion beats.

Probably the cardiac ballet is more likely to occur when the myocardium is damaged. Of the 7 patients described in this paper, the condition occurred during an operation for mitral stenosis in Case 1. The other 6 patients were seriously or dangerously ill. Five of these 6 patients had clinical manifestations of ischaemic heart disease (Cases 2, 3, 4, 5, and 7); the sixth (Case 6) suffered from severe cor pulmonale and had no known history of ischaemic heart disease, but substantial coronary atherosclerosis was found at necropsy. Hypokalaemia and atrial tachycardia with second-degree atrioventricular block was present in Case 4; digitalis therapy had been discontinued before admission to hospital, but digitalis toxicity was possible. Digitalis toxicity was also possible in Cases 6 and 7.

The association of the $R$ on $T$ phenomenon with cardiac ballet is unlikely to be accidental, for $T$ wave interruptions occurred in all 7 examples of this arrhythmia. The occurrence of $R$ waves interrupting $T$ waves has a relation with the occurrence of sudden death (Smirk, 1949; Smirk and Palmer, 1960; Lawrie et al., 1967; Laham, 1967). R waves interrupting $T$ waves often herald ventricular tachyarrhythmias, and are frequently found at the onset of, or during, paroxysmal ventricular tachycardia, and at the start of ventricular flutter and ventricular 


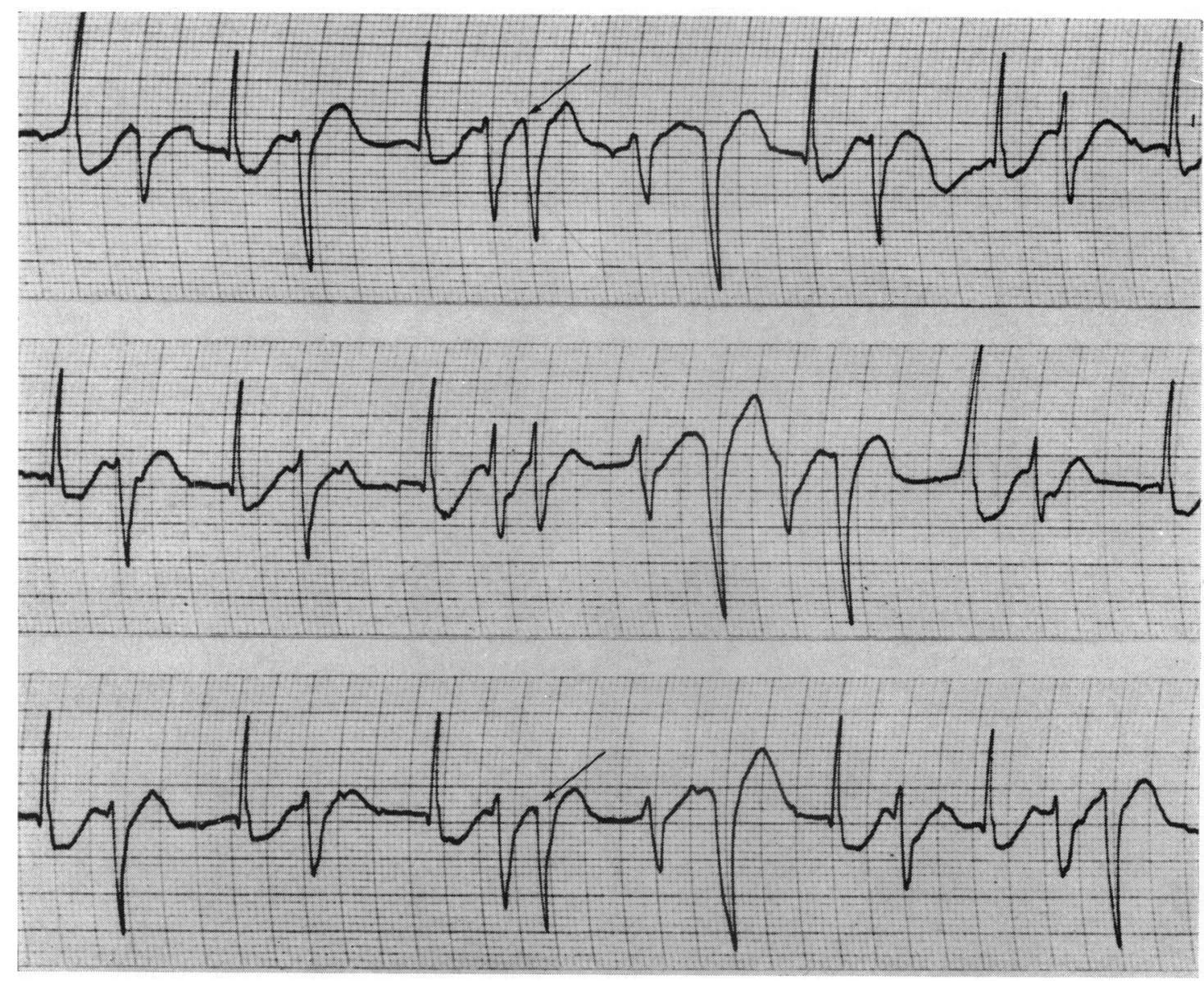

Fig. 7.-Case 7. The three traces are of a cube system scalar lead A record, and were obtained in descending order of traces. The upper and lower traces are similar especially from their fifth to tenth ventricular complexes. The middle trace resembles the other two traces in the general sequence of ventricular complexes, but the sixth, seventh, and tenth ventricular complexes differ in configuration. Two examples of $T$ waves which show early interruption by ventricular ectopic complexes are marked by arrows.

fibrillation. These features of the $R$ on $T$ phenomenon have been established clinically (Smirk, 1945, 1949; Schmidt, 1952; Katz and Pick, 1956b; Smirk and Palmer, 1960; Brown et al., 1963; Julian, Valentine, and Miller, 1964; Pick, 1964; Sowton, 1964; Sowton, Leatham, and Carson, 1964; Ahuja, Gutierrez, and Manning, 1966; Cohen, 1966; Surawicz and Zumino, 1966; Dolara, 1967; Lown et al., 1967; Restieaux et al., 1967; Gutierrez, Changfoot, and Peretz, 1968; Ng, 1968), and experimentally (Smirk, 1945; Chenoweth, 1946; Fastier and Smirk, 1948; Garb and Chenoweth, 1948; Fastier, 1951 ; Preston, McFadden, and Moe, 1959; Moore and Swain, 1960a, b; Smirk, Nolla-Panades, and Wallis, 1964; Carroll, Ahuja, and Manning, 1965).

Of the 7 patients described in this paper, short runs of ventricular tachycardia were found in 6 (Cases 1, 2, 3, 5, 6, and 7), though not always at the time of the cardiac ballet. Ventricular fibrillation was recorded in 2 of the patients (Cases 2 and 7) who had died suddenly, one (Case 2) having developed repetitive electrocardiographic sequences which directly preceded an episode of ventricular fibrillation. Four of the 7 patients died during the hospital admission in which they showed the cardiac ballet rhythm, and 1 other patient died 5 months after discharge from hospital.

All this raises the question whether the cardiac ballet rhythm has additional adverse prognostic significance over and above that of the $R$ on $T$ phenomenon. More observation is required, but the question may well be answered in the affirmative for at least some types of this abnormal rhythm. 


\section{SUMMARY}

Seven cases are described of an arrhythmia which we have named the "cardiac ballet". It consists of a sequence of multiform ventricular complexes sometimes interspersed with ventricular complexes of the contour of sinus beats, forming a pattern which repeats itself. The $R$ on $T$ phenomenon was found in all the patients who had this arrhythmia. Five of the patients died; two were found to have died suddenly from ventricular fibrillation. The cardiac ballet rhythm directly preceded an episode of ventricular fibrillation which was recorded in one of these cases of sudden death.

We are grateful to Mr. A. T. Wallis for help with the recordings. Thanks are due to the Medical Research Council of New Zealand for financial support.

\section{REFERENCES}

Ahuja, S. P., Gutierrez, M. R., and Manning, G. W. (1966). Mode of onset of ventricular arrhythmias. In Abstracts, Part II, The V World Congress of Cardiology, New Delhi, p. 4. National Printing Works, Delhi.

Brown, K. W. G., MacMillan, R. L., Forbath, N., Mel'grano, F., and Scott, J. W. (1963). Coronary unit. An intensive-care centre for acute myocardial infarction. Lancet, 2, 349.

Carroll, S. E., Ahuja, S. P., and Manning, G. W. (1965). The initiation of ventricular tachycardia and fibrillation in experimental coronary artery occlusion. Amer. $\mathcal{F}$. Cardiol., 16, 813.

Chenoweth, M. B. (1946). Ventricular fibrillation induced by hydrocarbons and epinephrine. f. industr. Hyg., 28, 151 .

Cohen, J. (1966). Paroxysmal tachycardia precipitated by atrial or ventricular premature systoles. In Mechanisms and Therapy of Cardiac Arrhythmias: The Fourteenth Hahnemann Symposium, p. 302 . Ed. by L. S. Dreifus and W. Likoff. Grune and Stratton, New York and London.

Dolara, A. (1967). Early premature ventricular beats, repetitive ventricular response, and ventricular fibrillation. Amer. Heart F., 74, 332.

Fastier, F. N. (1951). Electrocardiographic features of "adrenaline syncope". F. Physiol. (Lond.), 112, 359.

—, and Smirk, F. H. (1948). Some properties of amarin, with special reference to its use in conjunction with adrenaline for the production of idio-ventricular rhythms. f. Physiol. (Lond.), 107, 318.

Garb, S., and Chenoweth, M. B. (1948). Studies on hydrocarbon-epinephrine induced ventricular fibrillation. $\mathcal{F}$. Pharmacol. exp. Ther., 94, 12.

Grishman, A., and Scherlis, L. (1952). Spatial Vectorcardiography. Saunders, Philadelphia.

Gutierrez, M. R., Changfoot, G. H., and Peretz, D. I. (1968). Significance of $T$ wave interruption by premature beats as a cause of sudden death. Canad. med. Ass. F., 98, 144.

Julian, D. G., Valentine, P. A., and Miller, G. G. (1964). Disturbances of rate, rhythm and conduction in acute myocardial infarction. Amer. F. Med., 37, 915.
Katz, L. N., and Pick, A. (1956a). Clinical Electrocardiography. Part I. The Arrhythmias, p. 370. Lea and Febiger, Philadelphia.

-, and - (1956b). Clinical Electrocardiography. Part I. The Arrhythmias, pp. 177, 368, and 510. Lea and Febiger, Philadelphia.

Laham, J. (1967). Le phénomène du " $R$ sur $T$ " (à propos de 25 observations personnelles). France méd., 30, 461.

Lawrie, D. M., Greenwood, T. W., Goddard, M., Harvey, A. C., Donald, K. W., Julian, D. G., and Oliver, M. F. (1967). A coronary-care unit in the routine management of acute myocardial infarction. Lancet, 2, 109.

Lown, B., Fakhro, A. M., Hood, W. B., Jr., and Thorn, G. W. (1967). The coronary care unit. New perspectives and directions. F. Amer. med. Ass., 199, 188.

Moore, J. I., and Swain, H. H. (1960a). Sensitization to ventricular fibrillation. I. Sensitization by a substituted propiophenone, U-0882. F. Pharmacol. exp. Ther., 128, 243.

$\longrightarrow$, and $-(1960 \mathrm{~b})$. Sensitization to ventricular fibrillation. II. Sensitization by amarine and congeners of U-0882. F. Pharmacol. exp. Ther., 128, 253.

$\mathrm{Ng}$, J. (1968). Interruption of $\mathrm{T}$ waves by intrinsic QRS complexes. N.Z. med. F., 67, 361 .

Palmer, D. G. (1962). Interruption of T waves by premature QRS complexes and the relationship of this phenomenon to ventricular fibrillation. Amer. Heart f., 63, 367.

Parkinson, J., and Papp, C. (1947). Repetitive paroxysmal tachycardia. Brit. Heart f., 9, 241.

Pick, A. (1964). Manifestations of a vulnerable phase in the human heart. In Sudden Cardiac Death, p. 44. Ed. by B. Surawicz and E. D. Pellegrino. Grune and Stratton, New York.

Preston, J. B., McFadden, S., and Moe, G. K. (1959). Atrioventricular transmission in young mammals. Amer. F. Physiol., 197, 236.

Restieaux, N., Bray, C., Bullard, H., Murray, M., Robinson, J., Brigden, W., and McDonald, L. (1967). 150 patients with cardiac infarction treated in a coronary unit. Lancet, 1, 1285.

Schmidt, W. (1952). Kammer-Extrasystolen äusserster Vorzeitigkeit als Vorläufer Morgagni-Adams-Stokesscher Anfälle. Z.Kreisl.-Forsch., 41, 590.

Smirk, F. H. (1945). Ventricular rhythm. Communication to the Royal Australasian College of Physicians.

(1949). R waves interrupting T waves. Brit. Heart F., 11, 23.

-, Nolla-Panades, J., and Wallis, T. (1964). Experimental ventricular flutter and ventricular paroxysmal tachycardia. Amer. F. Cardiol., 14, 79.

—, and Palmer, D. G. (1960). A myocardial syndrome with particular reference to the occurrence of sudden death and of premature systoles interrupting antecedent T waves. Amer. F. Cardiol., 6, 620.

Sowton, E. (1964). The use of artificial pacemaking in cardiac resuscitation. Proc. roy. Soc. Med., 57, 368.

—, Leatham, A., and Carson, P. (1964). The suppression of arrhythmias by artificial pacemaking. Lancet, 2 , 1098.

Stock, J. P. P. (1962). Repetitive paroxysmal ventricular tachycardia. Brit. Heart f., 24, 297.

Surawicz, B., and Zumino, A. P. (1966). The vulnerable period of ventricular excitation. In Mechanisms and Therapy of Cardiac Arrhythmias, p. 255. Ed. by L. S. Dreifus and W. Likoff. Grune and Stratton, New York and London.

Wallis, A. T., Meek, A. P., and Ng, J. (1968). Clinical and experimental applications of a central recording system (in the Wellcome Medical Research Institute, University of Otago Medical School). N.Z. med. F., 67, 356. 